'Sala de Ejercicio Físico y Salud, Hospital de Los Lagos, Chile.

${ }^{2}$ Departamento Ciencias de la Actividad Física, Universidad de Los Lagos, Osorno, Chile. ${ }^{3}$ Facultad de Educación, Ciencias Sociales y Humanidades,

Departamento de Educación Física, Universidad de la Frontera, Temuco, Chile. ${ }^{4}$ Centro de Salud Familiar "Tomás Rojas" de Los Lagos, Chile. ${ }^{a}$ Kinesiólogo.

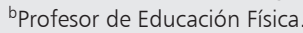
CPhD.

${ }^{\mathrm{d}} \mathrm{MSc}$.

Financiamiento: Este proyecto de investigación fue financiado con fondos del hospital público de la comuna de Los Lagos, con fondos del Centro de Salud Familiar "Tomás Rojas" de la comuna de Los Lagos, con fondos del programa comunal de promoción de la salud, pertenecientes a la SEREMI de Salud de la Región de Los Ríos y con fondos de aportes privados. No se declaran conflictos de interés entre las fuentes de financiamiento en el diseño del presente estudio.

Recibido el 18 de marzo de 2015 aceptado el 16 noviembre de

2015.

Correspondencia a: Cristian Álvarez MSc. Prat \#162 Centro de Salud Familiar "Tomás Rojas" de Los Lagos, Región de Los Ríos, Chile. profecristian.alvarez@gmail.com

\section{Interacción entre farmacoterapia hipotensiva y terapia con ejercicio físico requiere regulación farmacológica en pacientes hipertensos}

\author{
JOHNATTAN CANO-MONTOYA ${ }^{1, a, d}$, RODRIGO RAMÍREZ-CAMPILLO ${ }^{2, b, c}$, \\ CRISTIAN MARTÍNEZ ${ }^{3, b, d}$, FARID SADE-CALLES ${ }^{4}$, \\ ANDRÉS SALAS-PARADA ${ }^{1}$, CRISTIAN ÁLVAREZ ${ }^{4, b, d}$

\section{Interaction between antihypertensive therapy and exercise training therapy requires drug regulation in hypertensive patients}

\begin{abstract}
Background: Exercise training could interact with the pharmacological therapy of hypertension, increasing the effects of these medications. Aim: To assess the effects of 12 weeks of physical training on blood pressure of hypertensive or diabetic patients, already receiving pharmacological therapy. Material and methods: Twelve participants with diabetes and hypertension, 16 participants with hypertension and 18 healthy participants were studied. During six weeks no intervention was carried out, except the treatment for their underlying conditions. During the ensuing six weeks, participants were subjected 12 sessions of physical training. Blood pressure was measured at baseline and after six and twelve weeks of intervention. Results: During the period without physical training, no changes in blood pressure were observed. After the physical training intervention systolic blood pressure decreased by 16, 17 and $20 \mathrm{~mm} \mathrm{Hg}$ in participants with diabetes and hypertension, participants with hypertension and healthy participants, respectively. Diastolic blood pressure decreased by 9 and $6 \mathrm{mmHg}$ in participants with diabetes and hypertension and hypertension alone, respectively. Conclusions: There is a decrease in blood pressure among hypertensive participants receiving pharmacological therapy, after a period of physical training.
\end{abstract}

(Rev Med Chile 2016; 144: 152-161)

Key words: Blood pressure; Hypertension; Physical exercise; Therapy.
L a hipertensión arterial (HTA) es una enfermedad de etiología desconocida que produimportantes consecuencias cardiovascutas causas de muerte ${ }^{2}$, donde el ejercicio físico es piedra angular en su tratamiento ${ }^{3,4}$. En casos de HTA, las guías clínicas recomiendan en primera instancia el "cambio de estilo de vida", donde en caso de no alcanzar los valores de meta terapéutica $(<140 / 90 \mathrm{mmHg})$, cada paciente tiene la posibilidad de acceder a una garantizada farmacoterapia, de acuerdo a las garantías explícitas de salud de Chile (GES) ${ }^{5}$.

La farmacoterapia ha generado efectos benéficos en la compensación y mantención de los niveles de enfermedad en pacientes con $\mathrm{HTA}^{2,6}$, aunque por otra parte, mediante la terapia con ejercicio se han comunicado resultados favora- 
bles en la normalización fisiológica de la presión arterial ${ }^{7-11}$, pero existen pocos reportes que han estudiado la interacción entre farmacoterapia hipotensiva versus ejercicio físico combinado en pacientes con $\mathrm{HTA}^{12,13}$.

Tanto una sesión de ejercicio aeróbico ${ }^{14}$ como de fuerza ${ }^{8}$ reducen la presión arterial entre $8 \mathrm{y}$ $24 \mathrm{~h}^{15,16}$, aunque después de programas prolongados, es frecuente encontrar mayores valores de reducción ${ }^{17,18}$. Poco se conoce acerca de los efectos después de una sesión única de ejercicio físico combinado utilizando high-intensity interval training (HIIT) y ejercicio de fuerza en pacientes con HTA, mucho menos los efectos de programas prolongados ( $\geq 4$ semanas). Conociéndose que una sesión única de ejercicio físico modifica la presión arterial en personas sanas ${ }^{14}$ e hipertensas $^{15,19}$, el incremento de programas regulares de ejercicio físico en la atención primaria, y a la no clara aún interacción entre farmacoterapia hipotensiva versus ejercicio físico en pacientes con $\mathrm{HTA}^{12}$, el primer objetivo de este estudio fue evaluar los efectos de 12 sesiones de ejercicio físico combinado en pacientes hipertensos sometidos a terapia farmacológica. Un segundo objetivo fue analizar los cambios en la presión arterial antes y después de cada sesión.

\section{Métodos}

En un estudio experimental y de corte trasversal, mediante llamado público, acudieron cincuenta sujetos adultos con diagnóstico de HTA, diabetes mellitus tipo 2 (DT2) o sanos, quienes fueron invitados a participar de un programa de ejercicio físico combinado y monitoreado durante 12 sesiones de intervención (6 semanas). Los participantes fueron asignados a uno de tres grupos: diabéticos tipo $2+$ hipertensión (DT2/HTA, $\mathrm{n}=12)$, hipertensión arterial (HTA, $\mathrm{n}=16)$ o a un grupo de sujetos sanos (SANOS, $\mathrm{n}=18$ ). La muestra final y análisis estadísticos se realizaron de acuerdo a los sujetos que finalizaron todos los procedimientos de intervención $(\mathrm{n}=46)$, descartándose 4 sujetos del grupo DT2/HTA (2 por enfermedad viral repentina y 2 por no cumplir con todas las evaluaciones).

Los criterios de inclusión fueron: Personas sedentarias ( $<150 \mathrm{~min}$ de actividad física/semana); IMC entre 25 y $39,9 \mathrm{~kg} / \mathrm{m}^{2}$; hipertenso en control y tratamiento farmacológico; diabético tipo 2 e hipertenso en tratamiento farmacológico. Definimos a un paciente hipertenso de acuerdo a la clasificación de la Joint National Committee on Prevention, Detection, Evaluation, and Treatment of High Blood Pressure: $\mathrm{PAS} / \mathrm{PAD}<120 / 80=$ "normal", 120-129/80-84 a 130-139/85-89= "pre hipertensión", $\geq 140 / 90$ = "hipertensión", 140-159/9099 = "hipertensión etapa 1", 160-179/100-109 a $\geq 180 / 110$ = "hipertensión etapa 2". Se reclutaron personas sanas, de edad, género y grado de actividad similar a los grupos estudiados, para formar el grupo de control.

Los criterios de exclusión fueron: Padecer insuficiencia hepática, presencia de obesidad mórbida, arritmia cardiaca, retinopatía, nefropatía, úlcera varicosa y tener enfermedad pulmonar obstructiva crónica.

Los pacientes con HTA y DT2 se encontraban con control actualizado en el programa de salud cardiovascular del Centro de Salud Familiar “Tomás Rojas” de Los Lagos. Antes del inicio, los pacientes firmaron un consentimiento informado, desarrollándose este trabajo considerando la Declaración de Helsinki y fue aprobado por el comité de ética del mismo centro de salud.

\section{Terapia farmacológica del grupo HTA}

Los pacientes con HTA se encontraban bajo tratamiento farmacológico, utilizando; a) antagonistas de la angiotensina II (losartan); b) inhibidores de la enzima convertidora de angiotensina (enalapril); c) inhibidores de la entrada a las células de los iones calcio a través de los canales lentos (nitrendipino); d) diuréticos de asa (furosemida); ye) fármacos salicilados antiinflamatorios $\mathrm{y}$ analgésicos (aspirina).

\section{Terapia farmacológica del grupo HTA/DT2}

Estos pacientes se encontraban utilizando hipoglicemiante oral (metformina) y por su condición de hipertensos; estaban bajo uno o más de los fármacos declarados en el grupo HTA. Dos pacientes (1 del grupo DT2/HTA y 1 del grupo HTA) padecían hipotiroidismo. Las dosis de farmacoterapia se declaran en unidades/día, no reportándose el horario de su ingesta y son presentados en la Tabla 1.

\section{Evaluación de la composición corporal}

La masa corporal y la talla se midió con una 
Tabla 1. Características generales de los pacientes intervenidos durante 12 sesiones con ejercicio físico combinado

\begin{tabular}{|c|c|c|c|c|c|}
\hline Variable & Test & $\begin{array}{l}\text { DT2/HTA } \\
(n=12)\end{array}$ & $\begin{array}{c}\text { HTA }^{B} \\
(n=16)\end{array}$ & $\begin{array}{l}\text { SANOSC } \\
(n=18)\end{array}$ & $\mathbf{P}_{\text {interaction }}$ \\
\hline Edad (años) & & $63 \pm 10$ & $54 \pm 15$ & $41 \pm 15^{A B}$ & 0,050 \\
\hline \multicolumn{6}{|l|}{ Antropometría } \\
\hline Peso $(k g)$ & $\begin{array}{l}\text { Pre }_{\text {EF0 }} \\
\text { Post }_{\text {EF6 }}\end{array}$ & $\begin{array}{l}83,6 \pm 9 \\
82,6 \pm 3\end{array}$ & $\begin{array}{l}79,7 \pm 19 \\
79,1 \pm 5\end{array}$ & $\begin{array}{l}71,3 \pm 14 \\
70,3 \pm 7\end{array}$ & 0,209 \\
\hline Talla (m) & Pre $_{\text {EFo }}$ & $1,58 \pm 0,07$ & $1,55 \pm 0,08$ & $1,56 \pm 0,06$ & 0,663 \\
\hline $\operatorname{IMC}\left(\mathrm{kg} / \mathrm{m}^{2}\right)$ & $\begin{array}{l}\text { Pre }_{\text {EFo }} \\
\text { Post }_{\text {EF6 }}\end{array}$ & $\begin{array}{l}33,4 \pm 13 \\
33,0 \pm 8\end{array}$ & $\begin{array}{l}33,1 \pm 10 \\
32,9 \pm 7\end{array}$ & $\begin{array}{l}29,2 \pm 11^{A B} \\
28,8 \pm 9^{A B}\end{array}$ & $\begin{array}{l}0,004 \\
0,345\end{array}$ \\
\hline \multicolumn{2}{|c|}{ Farmacoterapia (dosis/rango unidades/frecuencia) } & Proporción & Proporción & & \\
\hline \multicolumn{2}{|c|}{ Metformina (850 mg/2 unidades/día) } & $12 / 12$ & 0/16 & & \\
\hline \multicolumn{2}{|c|}{ Glibenclamida (5 mg/1 unidad/día) } & $4 / 12$ & $0 / 16$ & & \\
\hline \multicolumn{2}{|c|}{ Enalapril (10 mg/ 1-3 unidades/día) } & $5 / 12$ & $8 / 16$ & & \\
\hline \multicolumn{2}{|c|}{ Losartan (50 mg/1-2 unidades/día) } & $5 / 12$ & $4 / 16$ & & \\
\hline \multicolumn{2}{|c|}{ Nitrendipino (20 mg/1 unidad/día) } & $4 / 12$ & $6 / 16$ & & \\
\hline \multicolumn{2}{|c|}{ Furosemida (40 mg/1 unidad/día) } & $2 / 12$ & $1 / 16$ & & \\
\hline \multicolumn{2}{|c|}{ Lovastatina (20 mg/1 unidad/día) } & $1 / 12$ & $1 / 16$ & & \\
\hline \multicolumn{2}{|c|}{ Atorvastatina (10-40 mg/1 unidad/día) } & $8 / 12$ & $2 / 16$ & & \\
\hline \multicolumn{2}{|c|}{ Gemfibrozilo (300 mg/1 unidad/día) } & $3 / 12$ & $0 / 16$ & & \\
\hline \multicolumn{2}{|c|}{ Atenolol (50 mg/1 unidad/día) } & $2 / 12$ & $2 / 16$ & & \\
\hline \multicolumn{2}{|c|}{ Propanolol (40 mg/1 unidad/día } & $2 / 12$ & $2 / 16$ & & \\
\hline \multicolumn{2}{|c|}{ Aspirina (500 mg/1 unidad/día) } & $8 / 12$ & $4 / 16$ & & \\
\hline \multicolumn{2}{|c|}{ Eutirox (75-100 mg/1 unidad/día) } & $1 / 12$ & $1 / 12$ & & \\
\hline
\end{tabular}

Grupos son descritos como; (DT2/HTA) Diabetes tipo 2 + Hipertensión, (HTA) Hipertensión y (SANOS) Pacientes sanos. Test se presentan como; $\left(\right.$ Pre $_{\mathrm{EFO}}$ ) Antes del programa de ejercicio físico combinado + farmacoterapia, (Post ${ }_{\mathrm{EF} 6}$ ) Después de 6 semanas de ejercicio físico combinado + farmacoterapia.

balanza de palanca en un estadiómetro profesional marca DETECTO ${ }^{\curvearrowleft}$ (modelo 3P7044, Webb City, USA), con precisión de $0,1 \mathrm{~cm}$. El IMC se calculó en base a la masa corporal dividida por la talla al cuadrado $\left(\mathrm{kg} / \mathrm{m}^{2}\right)$.

\section{Evaluación de la presión arterial}

Siguiendo criterios ampliamente utilizados ${ }^{2}$, la presión arterial sistólica y diastólica se midieron con un monitor digital marca OMRON ${ }^{\circledR}$ (modelo HEM-742INT, USA) entre las 10:00 y 12:00 h y entre las 14:00 y 16:00 h, durante tres oportunidades en el brazo izquierdo, en posición sentado después de 15 min de reposo, desarrollándose ello, antes del período de familiarización $\left(\right.$ Pre $\left._{\mathrm{F}}\right)$, antes de la intervención $\left(\operatorname{Pre}_{\mathrm{EF} 0}\right)$, antes $\left(\operatorname{Pre}_{\mathrm{S}}\right)$ y después de 15 min de cada sesión (Post15min), y después de intervención ( Post $\left._{\mathrm{EF} F}\right)$.

\section{Sesiones de adaptación}

Se realizaron 4 sesiones de familiarización, utilizando bicicletas estáticas marca EVERLAST ${ }^{\circledR}$ (modelo YC4620, USA), realizando $20 \mathrm{~min}$ de pedaleo a baja intensidad (1-3 en la escala de Borg modificada de $1-10$ puntos) ${ }^{21} y \leq 70 \%$ de su frecuencia cardiaca máxima y adicionalmente realizaron 3 ejercicios de fuerza.

\section{Programa de ejercicio físico combinado}

Durante el HIIT se completaron 8-14 intervalos/sesión, a intensidad $80-100 \%$ de la frecuencia cardiaca máxima (FCM), equivaliendo ello a una carga en bicicleta entre 8 y 10 puntos de la escala de Borg modificada de 1-10 puntos ${ }^{21}$. Cada intervalo consistió en 1 min de pedaleo en bicicleta, seguido por 2 min de pausa inactiva similar a protocolos previos $^{22}$. 
Para el ejercicio de fuerza se utilizaron pesos libres, y se ejercitaron 3 grupos musculares con los ejercicios; curl de bíceps, extensión vertical de tríceps y remo con mancuernas para músculos de la espalda. Se realizó 1 min de ejercicio hasta alcanzar la "fatiga muscular", seguido por $1 \mathrm{~min}$ de descanso inactivo y repetido en 3 series, método denominado $1 \times 1 \times 3$ y utilizado recientemente en pacientes mujeres con obesidad ${ }^{23}$. Se alcanzó la fatiga muscular entre 20 y $40 \%$ de 1 repetición máxima (1RM). Las sesiones se realizaron entre 10 y $12 \mathrm{~h}$ y entre las 14 y 16 h, siendo su duración $\leq 60 \mathrm{~min}$. El programa se desarrolló en la sala de ejercicio del Hospital de Los Lagos. La adherencia al programa fue de DT2/HTA 95\%, HTA 95\% y SANOS 93\%.

\section{Análisis estadístico}

Se utilizó media y $( \pm)$ desviación estándar para describir las variables. Se utilizó el test de Shapiro-Wilk para determinar la normalidad y el test de Levene para conocer la homocedasticidad. Se utilizó el análisis ANOVA de una vía para determinar diferencias en la línea de base, aplicándose también un análisis de medidas repetidas (grupos $\mathrm{x}$ tiempo) para ver diferencias pre-post. El nivel de error alfa se estableció en $\mathrm{p}<0,05$. Los análisis se realizaron utilizando el software SPSS (versión 18.0).

Tabla 2. Modificación de la presión arterial; durante un período de farmacoterapia (A), durante un período de familiarización al ejercicio (B), y después de un período de farmacoterapia + ejercicio físico combinado (C)

\begin{tabular}{|c|c|c|c|c|c|c|}
\hline Variable & Test & Momento & $\begin{array}{l}\text { DT2/HTA } \\
(n=12)\end{array}$ & $\begin{array}{c}\text { HTA } \\
(n=16)\end{array}$ & $\begin{array}{l}\text { SANOS } \\
(n=18)\end{array}$ & $\boldsymbol{P}_{\text {interaction }}$ \\
\hline \multicolumn{7}{|c|}{$\begin{array}{l}\text { (A) Período de Farmacoterapia } \\
\text { (6 semanas) }\end{array}$} \\
\hline PAS (mmHg) & $\begin{array}{l}\text { Pre }_{\mathrm{Ft0}} \\
\text { Post }_{\mathrm{Ft} 6}\end{array}$ & & $\begin{array}{l}149 \pm 20 \\
147 \pm 26\end{array}$ & $\begin{array}{l}147 \pm 12 \\
145 \pm 10\end{array}$ & $\begin{array}{l}134 \pm 12 \\
131 \pm 15\end{array}$ & $\begin{array}{l}0,147 \\
0,131\end{array}$ \\
\hline $\mathrm{PAD}(\mathrm{mmHg})$ & $\begin{array}{l}\text { Pre }_{\mathrm{Ft0}} \\
\text { Post }_{\mathrm{Ft} 6}\end{array}$ & & $\begin{array}{l}88 \pm 10 \\
89 \pm 8\end{array}$ & $\begin{array}{l}81 \pm 12 \\
83 \pm 10\end{array}$ & $\begin{array}{l}84 \pm 9 \\
82 \pm 13\end{array}$ & $\begin{array}{l}0,389 \\
0,432\end{array}$ \\
\hline \multicolumn{7}{|c|}{$\begin{array}{l}\text { (B) Período de Familiarización }{ }^{F} \\
\text { (2 semanas) }\end{array}$} \\
\hline $\begin{array}{l}\text { PAS }(\mathrm{mmHg}) \\
\operatorname{PAD}(\mathrm{mmHg})\end{array}$ & $\begin{array}{l}\text { Pre }_{F} \\
\text { Pre }_{F}\end{array}$ & & $\begin{array}{r}146 \pm 26 \\
87 \pm 9\end{array}$ & $\begin{array}{r}143 \pm 10 \\
83 \pm 8\end{array}$ & $\begin{array}{r}132 \pm 16 \\
83 \pm 8\end{array}$ & $\begin{array}{l}0,138 \\
0,117\end{array}$ \\
\hline \multicolumn{7}{|c|}{$\begin{array}{l}\text { (C) Período de Ejercicio Combinado + Farmacoterapia } \\
\text { (6 semanas) }\end{array}$} \\
\hline \multirow[t]{2}{*}{ PAS (mmHg) } & Pre $_{\text {EFo }}$ & $\begin{array}{l}\text { Pre }_{s} \\
\text { Post15min }\end{array}$ & $\begin{array}{l}135 \pm 17^{F} \\
132 \pm 13\end{array}$ & $\begin{array}{l}133 \pm 13^{F} \\
122 \pm 11^{*}\end{array}$ & $\begin{array}{l}120 \pm 12^{F} \\
118 \pm 14\end{array}$ & $\begin{array}{l}0,793 \\
0,659\end{array}$ \\
\hline & Post $_{\text {EF6 }}$ & $\begin{array}{l}\text { Pre }_{s} \\
\text { Post15min }\end{array}$ & $\begin{array}{l}130 \pm 8^{F} \\
115 \pm 9^{* \pi}\end{array}$ & $\begin{array}{l}126 \pm 9^{F} \\
115 \pm 6^{* \pi}\end{array}$ & $\begin{array}{l}112 \pm 15^{F} \\
114 \pm 11\end{array}$ & $\begin{array}{l}0,151 \\
0,986\end{array}$ \\
\hline \multirow[t]{2}{*}{$\mathrm{PAD}(\mathrm{mmHg})$} & Pre $_{\text {EFo }}$ & $\begin{array}{l}\text { Pre }_{s} \\
\text { Post15min }\end{array}$ & $\begin{array}{l}81 \pm 14^{F} \\
74 \pm 8^{*}\end{array}$ & $\begin{array}{l}77 \pm 13^{F} \\
76 \pm 12\end{array}$ & $\begin{array}{l}83 \pm 13 \\
78 \pm 14\end{array}$ & $\begin{array}{l}0,516 \\
0,546\end{array}$ \\
\hline & Post $_{\text {EF6 }}$ & $\begin{array}{l}\text { Pre }_{\mathrm{s}} \\
\text { Post15min }\end{array}$ & $\begin{array}{l}78 \pm 6^{F} \\
73 \pm 8^{*}\end{array}$ & $\begin{array}{l}77 \pm 6^{F} \\
71 \pm 7\end{array}$ & $\begin{array}{l}80 \pm 8 \\
75 \pm 8^{*}\end{array}$ & $\begin{array}{l}0,686 \\
0,603\end{array}$ \\
\hline
\end{tabular}

Grupos son descritos como; (DT2/HTA) Diabetes tipo 2 + Hipertensión, (HTA) Hipertensión y (SANOS) Pacientes sanos. Variables son descritas como; (PAS) Presión arterial sistólica, (PAD) Presión arterial diastólica. Test son descritos como; (Pre Fto $_{\text {) }}$ Antes de farmacoterapia, (Post ${ }_{\mathrm{Ft}}$ ) Después de 6 semanas de farmacoterapia, (Pre $\mathrm{F}_{\mathrm{F}}$ Antes del período de familiarización al ejercicio, $\left(\right.$ Pre $_{\mathrm{EFO}}$ ) Antes del programa de ejercicio físico combinado + farmacoterapia, (Post $\mathrm{EFF}_{\text {) }}$ Después de 6 semanas de ejercicio físico combinado + farmacoterapia, Momentos son descritos como; (Pres) Antes de una sesión de ejercicio, (Post15min) Después de 15 min de finalizado una sesión de ejercicio, Cambios son presentados como; ${ }^{\mathrm{F} C a m b i o s}$ significativos vs Pre $\mathrm{F}_{\mathrm{F}}$ nivel $\mathrm{p}<0,05$. ${ }^{*}$ Cambios significativos vs Pre $_{\mathrm{s}}$ a nivel $p<0,05$. "Cambios significativos vs Pre ${ }_{\mathrm{EF} 0}$ a nivel $\mathrm{p}<0,05$. 
Tabla 3. Cambios en la proporción de pacientes con diagnóstico de hipertensión, monitoreados antes del período de familiarización y después de 6 semanas de ejercicio físico combinado + farmacoterapia

\begin{tabular}{|c|c|c|c|}
\hline Período & $\begin{array}{l}\text { DT2/HTA } \\
(n=12)\end{array}$ & $\begin{array}{c}\text { HTA } \\
(n=16)\end{array}$ & $\begin{array}{l}\text { SANOS } \\
(n=18)\end{array}$ \\
\hline \multicolumn{4}{|l|}{ Antes del período de familiarización (sólo farmacoterapia) } \\
\hline $\mathrm{n}^{\circ}$ de pacientes con PA < 140/90 mmHg / (\% muestra) & $4 /(33 \%)$ & $4 /(25 \%)$ & $18 /(100 \%)$ \\
\hline $\mathrm{n}^{\circ}$ de pacientes con $\mathrm{PA} \geq 140 / 90$ mmHg / (\% muestra) & $8 /(66 \%)$ & $12 /(75 \%)$ & $0 / \quad(0 \%)$ \\
\hline \multicolumn{4}{|l|}{ Después de 6 semanas de ejercicio físico combinado + farmacoterapia } \\
\hline $\mathrm{n}^{\circ}$ de pacientes con PA < 140/90 mmHg / (\% muestra) & $11 /(91 \%)$ & $14 /(87 \%)$ & $18 /(100 \%)$ \\
\hline $\mathrm{n}^{\circ}$ de pacientes con PA $\geq 140 / 90 \mathrm{mmHg} /$ (\% muestra) & $1 /(8 \%)$ & $2 /(12 \%)$ & $0 / \quad(0 \%)$ \\
\hline Casos de hipotensión nocturna & $1 /(8 \%)$ & $3 /(18 \%)$ & $0 / \quad(0 \%)$ \\
\hline
\end{tabular}

Grupos son descritos como; (DT2/HTA) Diabetes tipo 2 + Hipertensión, (HTA) Hipertensión y (SANOS) Pacientes sanos. (PA) Presión arterial.

\section{Resultados}

Después de 6 semanas del período de farmacoterapia en los grupos DT2/HTA e HTA, no se presentaron modificaciones en la PAS y PAD (Tabla 2).

Se presentó una reducción significativa $(\mathrm{p}<0,05)$ en la PAS desde el período de familiarización $\left(\right.$ Pre $_{\mathrm{F}}$ ) hasta el término del programa $\left(\right.$ Post $\left._{\mathrm{EF} 6}\right)$ en los grupos DT2/HTA desde $146 \pm 26 \mathrm{a}$ $130 \pm 8 \mathrm{mmHg}$ y en el grupo HTA desde $143 \pm 10$ a $126 \pm 9 \mathrm{mmHg}$, así como en el grupo SANOS desde $132 \pm 16$ a $112 \pm 15 \mathrm{mmHg}$. Se registró una reducción significativa en la $\mathrm{PAD}$ desde el mismo período en el grupo DT2/HTA desde $87 \pm 9$ a $78 \pm 6 \mathrm{mmHg}$ y en el grupo HTA desde $83 \pm 8$ a $77 \pm 6 \mathrm{mmHg}$ (Tabla 2).

Se presentó un incremento en el porcentaje de pacientes compensados $(<140 / 90 \mathrm{mmHg})$ en el grupo DT2/HTA desde 33 a $91 \%$ y en el grupo HTA desde 25 a $87 \%$ (Tabla 3). Se registraron 4 casos de hipotensión nocturna, 1 caso en el grupo DT2/HTA y 3 casos en el grupo HTA (Tabla 3 ).

En los cambios en cada sesión única, se presentó una reducción significativa de la PAS en el grupo DT2/HTA entre las sesiones 5 a la 12 (Figura 1A), ocurriendo lo mismo en las sesiones 1, 5 y entre la 7 y 12 del grupo HTA (Figura 1B), y finalmente en las sesiones 2, 3, 6 y 10 del grupo SANOS (Figura 1C).

Se presentó una reducción significativa de la PAD en el grupo DT2/HTA en las sesiones 1, 6, 8, 9, 11 y 12 (Figura $2 \mathrm{~A}$ ), ocurriendo lo mismo des- pués de las sesiones 2, 4, y entre la 9, y 12 del grupo HTA (Figura 2B), y finalmente en las sesiones 10 y 12 del grupo SANOS (Figura 2C).

\section{Discusión}

Los hallazgos más relevantes del presente estudio indican que la interacción de 12 sesiones de farmacoterapia + ejercicio físico combinado reducen la presión sistólica (PAS) y diastólica (PAD) a niveles de compensación $(<140 / 90 \mathrm{mmHg})$ en pacientes con hipertensión (HTA), generándose una necesidad de regular la dosificación farmacológica en algunos pacientes con el fin de reducir los riesgos de hipotensión (Tabla 2).

En el período de farmacoterapia no se modificó la presión arterial, Tabla 2. Sin cambios importantes en el peso corporal (Tabla 1), después de ejercicio combinado + farmacoterapia, se incrementó la cantidad de pacientes con clasificación de presión arterial en compensación (PAS/PAD < 140/90 mmHg) (Tabla 3). Son conocidos los efectos de una sesión única ${ }^{14,16,24,25} \mathrm{y}$ de programas prolongados de ejercicio ${ }^{7-9,18,26}$, aunque reportan diferentes resultados. Después de 9 semanas de ejercicio (caminar, 3 sesiones/semana, 30/45 min, 50/70\% FCM), Miller y cols. ${ }^{27}$ reportaron una reducción de PAS - 9 y PAD $-5 \mathrm{mmHg}$. Por otra parte, durante ejercicio de fuerza de 8 semanas ( $80 \%$ de $1 \mathrm{RM})$, Tibana y cols., reportaron una reducción de PAS en $-8,8$ y $\mathrm{PAD}-7,8 \mathrm{mmHg}$. Debido a que algunos sujetos redujeron $-13 \mathrm{mmHg}$ y otros presentaron un incremento de $+11 \mathrm{mmHg}$, los autores de este 


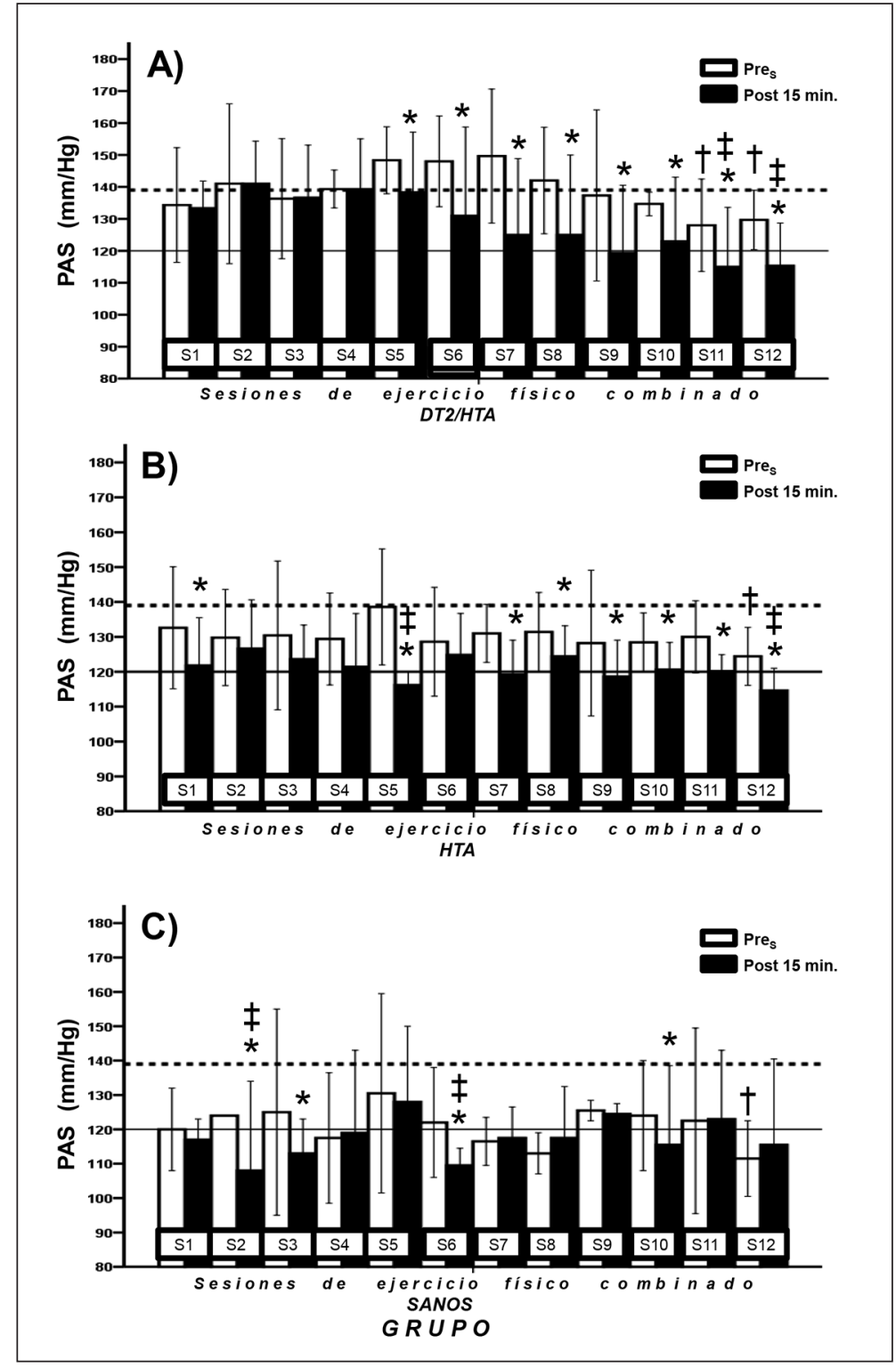

Figura 1. Cambios en la presión arterial sistólica posterior a 12 sesiones de ejercicio combinado + farmacoterapia. Variables se presentan como: (PAS) Presión arterial sistólica. (Pre $\mathrm{s}_{\mathrm{s}}$ Antes de una sesión de ejercicio, (Post15min) Después de 15 min de finalizado una sesión de ejercicio. Grupos se presentan como: (DT2/HTA) Diabetes tipo $2+$ Hipertensión, (HTA) Hipertensión y (SANOS) Pacientes sanos. (S1), (S2), (S3).... etc....indican el número de cada sesión de ejercicio físico combinado respectivamente. (---) Línea intermitente indica valores límites para "Hipertensión", (-) Línea continua indica valores límites para "normalidad". Cambios se presentan como: *Indica $\mathrm{p}<0,05$ entre (Pres) y (Post $15 \mathrm{~min}$ ). †Indica $p<0,05$ respecto a $\left(\right.$ Pre $\left._{s}\right)$ de sesión 1. łIndica $p<0,05$ respecto a (Post $15 \mathrm{~min}$.) de sesión 1 . estudio erróneamente concluyeron que el ejercicio prolongado no modifica la presión arterial.

Después de 15 semanas Cozza y cols. ${ }^{13}$ aplicando ejercicio continuo (45 min/sesión, $70-80 \%$ FCreserva) redujeron la PAS -6 y similarmente PAD -6 mmHg. Aunque los pacientes fueron hipertensos bajo farmacoterapia y ejercicio físico, al margen de los resultados, existen diferencias con nuestro estudio a nivel metodológico, como el tipo de ejercicio y tiempo invertido (bicicleta versus treadmill, 12 sesiones versus 45 sesiones), hechos claves cuando los pacientes son de edad avanzada, y cuando la principal excusa de la población para no hacer ejercicio es "no tengo tiempo" 28 .

Castaneda y cols. ${ }^{9}$, después de 16 semanas de ejercicio de fuerza (3 sesiones/semana, $45 \mathrm{~min} /$ sesión, 3 series/8 repeticiones [Rps], 60-80\% 1RM) en adultos mayores con HTA y DT2 bajo farmacoterapia hipotensiva e hipoglicemiante, reportaron una reducción en PAS de -9,7 y en PAD 


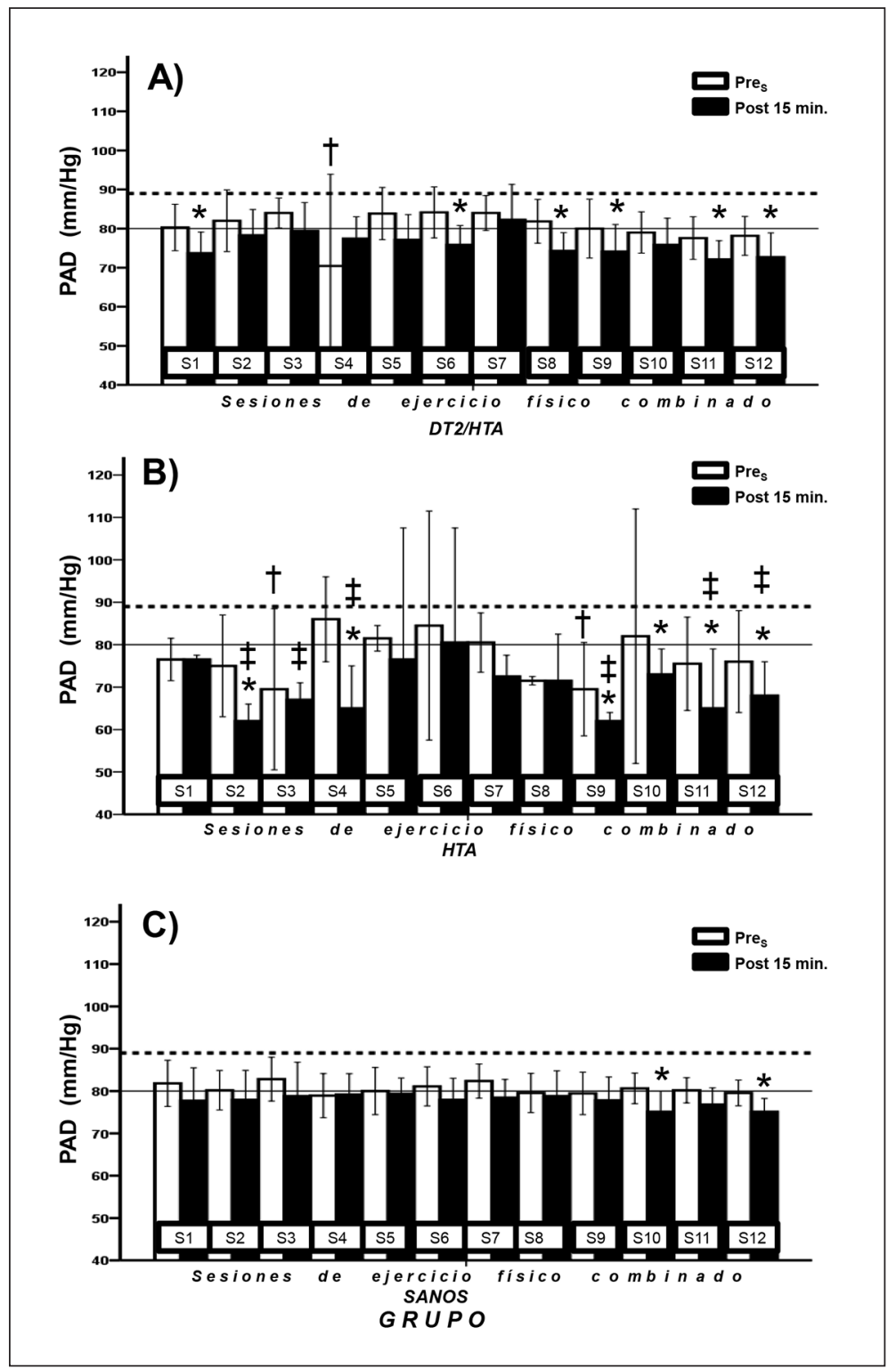

Figura 2. Cambios en la presión arterial sistólica posterior a 12 sesiones de ejercicio combinado + farmacoterapia. Variables se presentan como: (PAD) Presión arterial diastólica. $\left(\right.$ Pre $_{s}$ ) Antes de una sesión de ejercicio, (Post15min) Después de 15 min de finalizado una sesión de ejercicio. Grupos se presentan como: (DT2/HTA) Diabetes tipo $2+$ Hipertensión, (HTA) Hipertensión y (SANOS) Pacientes sanos. (S1), (S2), (S3)... etc...indican el número de cada sesión de ejercicio físico respectivamente. (---) Línea intermitente indica valores límites para "Hipertensión", (-) Línea continua indica valores límites para "normalidad". Cambios se presentan como: *Indica $\mathrm{p}<0,05$ entre $\left(\right.$ Pre $\left._{\mathrm{s}}\right)$ y (Post $15 \mathrm{~min}$ ). tIndica $p<0,05$ respecto a $\left(\right.$ Pre $\left._{s}\right)$ de sesión 1. łIndica $p<0,05$ respecto a (Post 15 min.) de sesión 1. de -3 mmHg. Bermudez y cols. ${ }^{29}$ aplicando ejercicio de fuerza (40\% de 1RM, 10 ejercicios, 3 series, 23 Rps) en sujetos normotensos, no encontraron cambios en la presión arterial. Por otra parte, Van Hoof y cols. ${ }^{26}$ posterior a 4 meses de ejercicio de fuerza (3 sesiones/semana, 4-12 Rps, 70-90\% de 1RM) similarmente, no reportaron cambios.

Claramente, existen pacientes "respondedores" y otros "no respondedores" 30 , similar a como reportaron Bouchard y cols. ${ }^{31}$, requiriéndose estudios para identificar los factores genéticos y ambientales que optimicen y ayuden a predecir los efectos del ejercicio. Nosotros encontramos que 3 pacientes no respondieron a la intervención (farmacoterapia + ejercicio físico) y no alcanzaron los valores de compensación en la presión arterial, siendo de interés para la ciencia, conocer también incluso los factores psico-socio-demográficos del por qué algunos pacientes pueden o no responder en mayor o menor grado. 
En las sesiones únicas de ejercicio, Melo y cols. ${ }^{25}$, aplicando una sesión de fuerza (6 ejercicios, 20 Rps, 40\% 1RM) en hipertensos bajo farmacoterapia, reportaron una reducción en PAS -12 y PAD -6 mmHg durante $10 \mathrm{~h}$. Hardy y Tucker ${ }^{24}$, en pacientes hipertensos, también en una sesión de fuerza generando fatiga muscular (7 ejercicios, 3 series, 8-12 Rps), redujeron la PAS -12 y PAD $-7 \mathrm{mmHg}$. En similares pacientes bajo farmacoterapia, Ciolac y cols. ${ }^{16}$ posterior a una sesión de ejercicio aeróbico (40 $\mathrm{min}, 60 \%$ de la FCreserva), redujeron la PAS -3 y PAD -2 mmHg hasta incluso $24 \mathrm{~h}$.

Una reciente revisión concluyó que tanto el ejercicio aeróbico como de fuerza mejoran la función endotelial ${ }^{32}$. Se han descrito como mecanismos responsables del efecto hipotensor, la reducción de la resistencia vascular periférica ${ }^{33}$, la angiogénesis ${ }^{34}$, el estrés por roce, y la producción de óxido nítrico ${ }^{11}$. En síntesis, durante sesiones únicas los reportes indican reducciones en PAS de -1 a -12 mmHg y en PAD de -2 a -7 $\mathrm{mmHg}{ }^{16,24,25,35}$, y considerando programas de larga duración reducciones en PAS de -8 a -10 y en PAD de -3 a $-8 \mathrm{mmHg}^{9,36}$. Tres estudios han propuesto que la duración y la magnitud del efecto hipotensor post ejercicio sería intensidad-dependiente ${ }^{35,37,38}$. Guimaraes y cols. ${ }^{38}$ mostraron que 16 semanas de HIIT y ejercicio aeróbico reducen la presión arterial similarmente, aunque sólo HIIT lograba reducir la rigidez arterial de pacientes con HTA.

Considerando la compensación de la presión arterial en los grupos DT2/HTA y HTA, y al reporte de hipotensión nocturna en 4 sujetos después del período de intervención (Tabla 3), es importante la adecuada derivación al profesional médico para regular la farmacoterapia hipotensora, sobre todo en aquellos pacientes que inician la ejercicio terapia con valores de clasificación normal $(\leq 120 / 80 \mathrm{mmHg})$ y que son adherentes.

En 104 pacientes, Cade y cols. ${ }^{12}$, después de 12 semanas de ejercicio (caminar 2 millas/día de moderada a vigorosa intensidad) debieron suspender la farmacoterapia hipotensora en 24 pacientes hipertensos quienes redujeron la PAS -22 y la PAD $-18 \mathrm{mmHg}$. Esta reducción trajo consigo adicionalmente una disminución en los costos farmacológicos diarios por paciente, desde $\$ 3,36$ a $\$ 0$ dólares. En aquellos sujetos en quienes sólo se debió regular la dosificación farmacológica $(\mathrm{n}=23)$, estos costos se redujeron desde $\$ 4,12$ a sólo \$ 2,52 dólares/día.

Nosotros encontramos reducciones de PAS - 16 a -20 mmHg y en PAD -3 a -9 mmHg (Tabla 2), incrementándose la cantidad de pacientes hipertensos bajo control en valores de compensación $(<140 / 90 \mathrm{mmHg}$ ) (Tabla 3). Esta reducción observada tiene implicancias clínicas relevantes, pues una reducción de $2 \mathrm{mmHg}$ en la PAS reduce la mortalidad por accidente cerebrovascular en $10 \%$ y por enfermedad cardiovascular en $7 \%{ }^{39}$.

Una fortaleza del presente estudio fue registrar durante 6 semanas previas la presión arterial cuando los pacientes sólo usaron farmacoterapia. Algunas debilidades fueron el que existieron diferencias en las edades de los grupos, así como también no se controlaron los patrones de actividad física ni dieta después de las sesiones, sin embargo se recordó a los participantes de mantener los mismos patrones declarados inicialmente.

Considerando el nuevo enfoque de salud familiar que fortalece la promoción de la salud y prevención de enfermedad ${ }^{40}$, junto con la elevada carga económica que genera la hipertensión y sus co-morbilidades asociadas ${ }^{41}$, es importante considerar la evaluación frecuente de la presión arterial y la dosificación farmacológica en pacientes quienes son también adherentes a programas de ejercicio físico, especialmente en pacientes hipertensos quienes presentan valores de normalidad antes de iniciar la sesión de ejercicio.

Agradecimientos: A todos los co-autores que hicieron posible que el estudio se llevara a cabo. A Carmen Flores, Directora del CESFAM “Tomás Rojas Vergara” de Los Lagos, por su incondicional apoyo en la promoción del ejercicio físico a nivel de atención primaria, y al personal del Hospital de Los Lagos.

\section{Referencias}

1. Coffman TM. Under pressure: the search for the essential mechanisms of hypertension. Nat Med 2011; 17 (11): 1402-9.

2. Chobanian AV, Bakris GL, Black HR, Cushman WC, Green LA, Izzo JL, et al. Seventh Report of the Joint National Committee on Prevention, Detection, Evaluation, and Treatment of High Blood Pressure. Hypertension 2003; 42 (6): 1206-52. 
3. Pescatello L. Exercise and hypertension: Recent advances in exercise prescription. Current Science Inc 2005; 7(4): 281-6.

4. Pescatello LS, Buckley T. Exercise guidelines for patients with high blood pressure: an update. J Cardiopulm Rehabil 1995; 15 (3): 239-40.

5. MINSAL. Guía clinica. Hipertensión Arterial Primaria o Escencial en Personas de 15 años y más. Santiago; 2010. p. 2-63.

6. The ALLHAT Officers Coordinators for the ALLHAT Collaborative Research Group. Major outcomes in high risk hypertensive patients randomized to angiotensin-converting enzyme inhibitor or calcium channel blocker vs diuretic: The Antihypertensive and Lipid Lowering treatment to prevent Heart Attack Trial (ALLHAT). JAMA 2002; 288 (23): 2981-97.

7. Blumenthal JA, Siegel WC, Appelbaum M. Failure of exercise to reduce blood pressure in patients with mild hypertension: Results of a randomized controlled trial. JAMA 1991; 266 (15): 2098-104.

8. Cardoso CG Jr, Gomides RS, Queiroz AC, Pinto LG, da Silveira Lobo F, Tinucci T, et al. Acute and chronic effects of aerobic and resistance exercise on ambulatory blood pressure. Clinics (Sao Paulo) 2010; 65 (3): 317-25.

9. Castaneda C, Layne JE, Munoz-Orians L, Gordon PL, Walsmith J, Foldvari M, et al. A Randomized Controlled Trial of Resistance Exercise Training to Improve Glycemic Control in Older Adults With Type 2 Diabetes. Diabetes Care 2002; 25 (12): 2335-41.

10. Pescatello LS, Miller B, Danias PG, Werner M, Hess $\mathrm{M}$, Baker C, et al. Dynamic exercise normalizes resting blood pressure in mildly hypertensive premenopausal women. Am Heart J 1999; 138 (5 Pt 1): 916-21.

11. Thijssen DHJ, Cable NT, Green DJ. Impact of exercise training on arterial wall thickness in humans. Clinical science (London, England: 1979) 2012; 122 (7): 311-22.

12. Cade R, Mars D, Wagemaker H, Zauner C, Packer D, Privette $\mathrm{M}$, et al. Effect of aerobic exercise training on patients with systemic arterial hypertension. Am J Med 1984; 77 (5): 785-90.

13. Cozza IC, Di Sacco THR, Mazon JH, Salgado MCO, Dutra SGV, Cesarino EJ, et al. Physical exercise improves cardiac autonomic modulation in hypertensive patients independently of angiotensin-converting enzyme inhibitor treatment. Hypertens Res 2012; 35 (1): 82-7.

14. Álvarez C, Olivo J, Robinson O, Quintero J, Carrasco V, Ramírez-Campillo R, et al. Respuesta hipotensiva de la presión sistólica y diastólica a una sesión de ejercicio aeróbico en niños, adolescentes y adultos. Rev Med Chile 2013; 141 (11): 1363-70.

15. Taylor-Tolbert NS, Dengel DR, Brown MD, McCole SD,
Pratley RE, Ferrell RE, et al. Ambulatory blood pressure after acute exercise in older men with essential hypertension. Am J Hypertens 2000; 13 (1 Pt 1): 44-51.

16. Ciolac EG, Guimarães GV, D’Ávila VM, Bortolotto LA, Doria EL, Bocchi EA. Acute aerobic exercise reduces 24-h ambulatory blood pressure levels in long-termtreated hypertensive patients. Clinics 2008; 63: 753-8.

17. Álvarez C, Ramírez-Campillo, R, Henríquez-Olguín, C, Castro-Sepúlveda, M, Carrasco, V, Martínez C. Eight weeks of combined high intensity intermitent exercise normalized altered metabolic parameters in women. Rev Med Chile 2014; 142: 458-66.

18. Álvarez C, Ramírez R, Flores M, Zúñiga C, Celis-Morales $\mathrm{CA}$. Efectos del ejercicio físico de alta intensidad y sobrecarga en parámetros de salud metabólica en mujeres sedentarias, pre-diabéticas con sobrepeso u obesidad. Rev Med Chile 2012; 140: 1289-96.

19. Terblanche E, Millen AE. The magnitude and duration of post-exercise hypotension after land and water exercises. Eur J Appl Physiol 2012; 112 (12): 4111-8.

20. O’Donovan G, Blazevich AJ, Boreham C, Cooper AR, Crank H, Ekelund U, et al. The ABC of Physical Activity for Health: a consensus statement from the British Association of Sport and Exercise Sciences. J Sports Sci 2010; 28 (6): 573-91.

21. Kendrick KR, Baxi SC, Smith RM. Usefulness of the modified 0-10 Borg scale in assessing the degree of dyspnea in patients with COPD and asthma. J Emerg Nurs 2000; 26 (3): 216-22.

22. Mancilla R, Torres P, Alvarez C, Schifferli I, Sapunar J, Diaz E. [High intensity interval training improves glycemic control and aerobic capacity in glucose intolerant patients]. Rev Med Chile 2014; 142 (1): 34-9.

23. Álvarez C, Campillo RR. Effects of a low intensity strength training program on overweight/obese and premenopausal/menopausal women. Revista Brasileira de Cineantropometria \& Desempenho Humano 2013; 15: 427-36.

24. Hardy DO, Tucker LA. The effects of a single bout of strength training on ambulatory blood pressure levels in 24 mildly hypertensive men. Am J Health Promot 1998; 13 (2): 69-72.

25. Melo CM, Alencar Filho AC, Tinucci T, Mion DJ, Forjaz CLM. Postexercise hypotension induced by low-intensity resistance exercise in hypertensive women receiving captopril. Blood Press Monit 2006; 11 (4): 183-9.

26. Van Hoof R, Macor F, Lijnen P, Staessen J, Thijs L, Vanhees L, et al. Effect of strength training on blood pressure measured in various conditions in sedentary men. Int J Sports Med 1996; 17 (6): 415-22.

27. Miller ER, Erlinger TP, Young DR, Jehn M, Charleston 
J, Rhodes D, et al. Results of the Diet, Exercise, and Weight Loss Intervention Trial (DEW-IT). Hypertension 2002; 40 (5): 612-8.

28. Anderson CB. When more is better: number of motives and reasons for quitting as correlates of physical activity in women. Health Educ Res 2003; 18 (5): 525-37.

29. Bermudes AMLdM, Vassallo DV, Vasquez EC, Lima EG. Monitorização ambulatorial da pressão arterial em indivíduos normotensos submetidos a duas sessões únicas de exercícios: resistido e aeróbio. Arquivos Brasileiros de Cardiologia 2004; 82: 57-64.

30. Davidsen PK, Gallagher IJ, Hartman JW, Tarnopolsky MA, Dela F, Helge JW, et al. High responders to resistance exercise training demonstrate differential regulation of skeletal muscle microRNA expression. J Appl Physiol 2011; 110 (2): 309-17.

31. Bouchard C, Blair SN, Church TS, Earnest CP, Hagberg JM, Häkkinen K, et al. Adverse metabolic response to regular exercise: is it a rare or common occurrence? PLoS One 2012; 7 (5): e37887.

32. Ashor A, Lara J, Siervo M, Celis-Morales C, Oggioni C, Jakovljevic D, et al. Exercise Modalities and Endothelial Function: A Systematic Review and Dose-Response Meta-Analysis of Randomized Controlled Trials. Sports Med 2014: 1-18.

33. Coats AJ, Conway J, Isea JE, Pannarale G, Sleight P, Somers VK. Systemic and forearm vascular resistance changes after upright bicycle exercise in man. J Physiol 1989; 413 (1): 289-98.

34. Brown MD, Hudlicka O. Modulation of physiological angiogenesis in skeletal muscle by mechanical forces: Involvement of VEGF and metalloproteinases. Angio- genesis 2003; 6 (1): 1-14.

35. Eicher JD, Maresh CM, Tsongalis GJ, Thompson PD, Pescatello LS. The additive blood pressure lowering effects of exercise intensity on post-exercise hypotension. Am Heart J 2010; 160 (3): 513-20.

36. Tibana RA, de Sousa NMF, da Cunha Nascimento D, Pereira GB, Thomas SG, Balsamo S, et al. Correlation between acute and chronic 24-hour blood pressure response to resistance training in adult women. Int J Sports Med 2015; 36 (1): 82-9.

37. Quinn TJ. Twenty-four hour, ambulatory blood pressure responses following acute exercise: impact of exercise intensity. J Hum Hypertens 2000; 14 (9): 547-53.

38. Guimaraes GV, Ciolac EG, Carvalho VO, D'Avila VM, Bortolotto LA, Bocchi EA. Effects of continuous vs. interval exercise training on blood pressure and arterial stiffness in treated hypertension. Hypertens Res 2010; 33 (6): 627-32.

39. Lewington S, Clarke R, Qizilbash N, Peto R, Collins R, Prospective Studies C. Age-specific relevance of usual blood pressure to vascular mortality: a meta-analysis of individual data for one million adults in 61 prospective studies. Lancet 2002; 360 (9349): 1903-13.

40. MINSAL. Orientaciones para la implementación del modelo de atención integral de salud familiar y comunitaria. Santiago: Subsecretaría de redes asistenciales; División de Atención Primaria; 2012.

41. Villarreal-Ríos E, Mathew-Quiroz A, Garza-Elizondo ME, Núñez-Rocha G, Salinas-Martínez AM, Gallegos-Handal M. Costo de la atención de la hipertensión arterial y su impacto en el presupuesto destinado a la salud en México. Salud Publica Mex 2002; 44: 7-13. 